\title{
CONGRUENCES AND GREEN'S RELATIONS ON REGULAR SEMIGROUPS
}

\author{
by T. E. HALL $\dagger$
}

(Received 10 December, 1970; revised 12 June, 1971)

1. Introduction. It is sometimes possible to reconstruct semigroups from some of their homomorphic images. Some recent examples have been the construction of bisimple inverse semigroups from fundamental bisimple inverse semigroups [9], and the construction of generalized inverse semigroups from inverse semigroups [12].

The success of this method depends on a knowledge of the similarities between the semigroup and its homomorphic image. We therefore examine in this paper the relationship between Green's relations on a regular semigroup and Green's relations on any homomorphic image. We now state the main specific question that we consider. Let $\mathscr{K}$ be any one of Green's relations $\mathscr{H}, \mathscr{L}, \mathscr{R}, \mathscr{D}$ and $\mathscr{J}$ and let $\rho$ be a congruence on a regular semigroup $S$.

QUESTION. If $A$ and $B$ are elements of $S / \rho$ that are $\mathscr{K}$-related in $S / \rho$, then are there elements $a \in A, b \in B$ such that $a$ and $b$ are $\mathscr{K}$-related in $S$ ?

We find that the answer is yes for $\mathscr{L}, \mathscr{R}$ and $\mathscr{D}$ and no for $\mathscr{H}$ and $\mathscr{J}$. Many related questions are considered. The partial analysis given leads to new proofs of many known results about congruences on regular semigroups.

In the final section semigroups other than regular semigroups are considered.

2. Preilminaries. We shall use whenever possible, and usually without comment, the notations of Clifford and Preston [1]. Further, for any semigroup $S$ we define a relation $\mathscr{V}$ by

$$
\mathscr{V}=\{(a, b) \in S \times S: a b a=a \text { and } b a b=b\} .
$$

We sometimes write $a \mathscr{V} b$ instead of $(a, b) \in \mathscr{V}$. We also write, for each element $a \in S$, $V(a)=\{b \in S:(a, b) \in \mathscr{V}\}$. Where there might otherwise be an ambiguity we shall denote Green's relations on a semigroup $S$ by $\mathscr{H}(S), \mathscr{L}(S)$, etc.

RESULT 1 [6, Lemma 2.2]. Let $\rho$ be a congruence on a regular semigroup $S$. Then each $\rho$-class that is an idempotent of $S / \rho$ contains an idempotent of $S$.

The following result, although trivial, gives rise to a determination of $\mathscr{L}$ on a regular semigroup that is often much easier to deal with than the definition of $\mathscr{L}$ for an arbitrary semigroup.

RESULT 2. Let $a$ and $b$ be elements of $a$ semigroup $S$ such that $a$ is a regular element. Then $L_{b} \leqq L_{a}$ if and only if $b=b a^{\prime} a$ for each [some] inverse $a^{\prime}$ of $a$.

Proof. (This is well known.) Suppose that $L_{b} \leqq L_{a}$. Then, for some element $x \in S^{1}$, $b=x a=x a a^{\prime} a=b a^{\prime} a$ for each [some] inverse $a^{\prime}$ of $a$, giving the "only if" statement. The "if" statement is trivial.

† This research was supported by a Nuffield Travelling Fellowship. 


\section{The relation $\mathscr{V}$.}

Lemma 1. (This was found independently by W. D. Munn.) Let $\rho$ be a congruence on a regular semigroup $S$ and let $A, B$ be any elements of $S / \rho$ such that $A \mathscr{V} B$ in $S / \rho$. Then there exist elements $a, b$ in $S$ such that $a \in A, b \in B$ and $a \mathscr{V} b$ in $S$.

Proof. Take any elements $x \in A, y \in B$ and let $(x y x y)^{\prime}$ be any inverse of $x y x y$ in $S$. Put $a=x y(x y x y)^{\prime} x y x, b=y(x y x y)^{\prime} x y$. Routinely one can show that $a \mathscr{V} b$ in $S$. Also, using $x \rho x y x$ and $y \rho y x y$ one obtains

$$
a=x y(x y x y)^{\prime} x y x \rho x y x y(x y x y)^{\prime} x y x y x=x y x y x \rho x
$$

and

$$
b=y(x y x y)^{\prime} x y \rho y x y x y(x y x y)^{\prime} x y x y=y x y x y \rho y .
$$

Hence $a \in A, b \in B$, giving the required result.

One can deduce Result 1 as a corollary as follows. Let $E$ be any idempotent of $S / \rho$. Since $E \mathscr{V} E$ in $S / \rho$, there exist $a \in E, a^{\prime} \in E$ such that $a \mathscr{V} a^{\prime}$ in $S$. Then $a a^{\prime} \in E E \subseteq E$ and $a a^{\prime}$ is an idempotent, giving Result 1.

Let us now consider again the statement and proof of Lemma 1. If $A$ is an idempotent of $S / \rho$, we can take $x$ to be an idempotent, and then we can show routinely that $a$ is also an idempotent. In general, when $A$ and $B$ satisfy the further condition of both being idempotents, there do not necessarily exist $a \in A, b \in B$ such that $a \mathscr{V} b, a^{2}=a, b^{2}=b$.

In the next theorem, the square brackets indicate an alternative reading.

THEOREM 2. Let $\rho$ be a congruence on a regular semigroup $S$ and let $n$ be a positive integer. If $A, B_{1}, B_{2}, \ldots, B_{n}$ are elements of $S / \rho$ such that $A \mathscr{V} B_{i}$, for $i=1,2, \ldots, n$, then there exist elements $a, b_{1}, b_{2}, \ldots, b_{n}$ in $S$ such that $a \in A, b_{i} \in B_{i}, a \mathscr{V} b_{i}$, for $i=1,2, \ldots, n$. Further, if $A\left[B_{1}\right]$ is an idempotent of $S / \rho$, then the element $a\left[b_{1}\right]$ can be taken to be an idempotent also.

Proof (by induction). We have proved already all the statements of the theorem for $n=1$. Suppose that all the statements are true for some positive integer $k<n$. Then there exist elements $x, y_{1}, y_{2}, \ldots, y_{k}$ in $S$ such that $x \in A, y_{i} \in B_{i}, x \mathscr{V} y_{i}$, for $i=1,2, \ldots, k$. Take any element $y_{k+1} \in B_{k+1}$. Let $\left(x y_{k+1} x y_{k+1}\right)^{\prime}$ be any inverse of $x y_{k+1} x y_{k+1}$ in $S$ and put $u=x y_{k+1}\left(x y_{k+1} x y_{k+1}\right)^{\prime} x y_{k+1} x, v_{k+1}=y_{k+1}\left(x y_{k+1} x y_{k+1}\right)^{\prime} x y_{k+1}$, and, for $i=1,2, \ldots, k$, put $v_{i}=y_{i} x y_{k+1}\left(x y_{k+1} x y_{k+1}\right)^{\prime} x y_{k+1} x y_{i}$. It is quite routine to show that $u \in A, v_{i} \in B_{i}, u \mathscr{V} v_{i}$, for $i=1,2, \ldots, k+1$.

If $A$ is an idempotent of $S / \rho$, then, by the inductive hypothesis, we can take $x$ to be an idempotent and then routinely we obtain that $u$ is an idempotent. Similar statements apply to $B_{1}, y_{1}$, and $v_{1}$. The theorem now follows by induction.

In general, when we have the further condition that $B_{1}, B_{2}$ are idempotents, it is not necessarily further possible that $b_{1}, b_{2}$ can be taken to be idempotents. Also, if $A_{1}, A_{2}, B_{1}, B_{2}$ are elements of $S / \rho$ such that $A_{i} \mathscr{V} B_{j}$, for $i, j=1,2$, there are not necessarily $a_{i} \in A_{i}, b_{j} \in B_{j}$ $(i, j=1,2)$ such that $a_{i} \mathscr{V} b_{j}(i, j=1,2)$.

COROLLARY 3. If $m$ is a positive integer and $S$ is a regular semigroup such that each element 
[idempotent] of $S$ has at most $m$ inverses, then the same is true of each homomorphic image of $S$.

In particular we have the first statement of Theorem 7.36 of [1], namely, that any homomorphic image of an inverse semigroup is also an inverse semigroup.

\section{Green's relation $\mathscr{L}$.}

LEMma 4. Let $\rho$ be a congruence on a regular semigroup $S$. Let $A_{1}, A_{2}, \ldots, A_{n}$ be any elements of $S / \rho$ such that $A_{1} \mathscr{L} A_{2} \mathscr{L} \ldots \mathscr{L} A_{n}$ in $S / \rho$. For $i=1,2, \ldots, n$, let $A_{i}^{\prime}$ be an inverse of $A_{i}$ in $S / \rho$. Then there exist elements $a_{1}, a_{2}, \ldots, a_{n}, a_{1}^{\prime}, a_{2}^{\prime}, \ldots, a_{n}^{\prime}$ in $S$ such that $a_{1} \mathscr{L} a_{2} \mathscr{L} \ldots \mathscr{L} a_{n}$ and such that, for $i=1,2, \ldots, n, a_{i} \in A_{i}, a_{i}^{\prime} \in A_{i}^{\prime}$ and $a_{i}^{\prime} \in V\left(a_{i}\right)$.

Proof (by induction). For $n=1$, the statement follows from Lemma 1. Suppose that the lemma is true for some positive integer $k<n$. Then there exist elements $x_{1}, x_{2}, \ldots, x_{k}$, $x_{1}^{\prime}, x_{2}^{\prime}, \ldots, x_{k}^{\prime}$ in $S$ such that $x_{1} \mathscr{L} x_{2} \mathscr{L} \ldots \mathscr{L} x_{n}$ in $S$ and such that $x_{i} \in A_{i}, x_{i}^{\prime} \in A_{i}^{\prime} \cap V\left(x_{i}\right)$, for $i=1,2, \ldots, k$. Take elements $x_{k+1}, x_{k+1}^{\prime}$ in $S$ such that $x_{k+1} \in A_{k+1}, x_{k+1}^{\prime} \in A_{k+1}^{\prime} \cap V\left(x_{k+1}\right)$ (Lemma 1). Let $y$ be any inverse of $x_{1}^{\prime} x_{1} x_{k+1}^{\prime} x_{k+1}$ and, for $i=1,2, \ldots, k+1$, put $u_{i}=$ $x_{l} x_{k+1}^{\prime} x_{k+1} y x_{1}^{\prime} x_{1}$ and put $u_{i}^{\prime}=x_{i}^{\prime} u_{i} x_{i}^{\prime}$. Now $x_{i} x_{j}^{\prime} x_{j}=x_{i}$ for $i, j=1,2, \ldots, k$ (Result 2) and from this it is routine to show that $u_{i}^{\prime} \in V\left(u_{i}\right)(i=1,2, \ldots, k+1)$; and also that $x_{i-1} x_{i}^{\prime} u_{i}=u_{i-1} \quad(i=2,3, \ldots, k+1), \quad x_{k+1} y x_{1}^{\prime} u_{1}=u_{k+1}$, whence $u_{1} \mathscr{L} u_{2} \mathscr{L} \ldots \mathscr{L} u_{k+1}$. Denote $y \rho$ by $Y$, an inverse of $A_{1}^{\prime} A_{1} A_{k+1}^{\prime} A_{k+1}=A_{1}^{\prime} A_{1}$ in $S / \rho$. Then, for $i=1,2, \ldots, k+1$, by Result 2,

$$
u_{i} \in A_{1} A_{k+1}^{\prime} A_{k+1} Y A_{1}^{\prime} A_{1}=A_{i} Y A_{1}^{\prime} A_{1}=A_{i} A_{1}^{\prime} A_{1} Y A_{1}^{\prime} A_{1}=A_{i} A_{1}^{\prime} A_{1}=A_{i}
$$

and

$$
u_{i}^{\prime}=x_{i}^{\prime} u_{i} x_{i}^{\prime} \in A_{i}^{\prime} A_{i} A_{i}^{\prime}=A_{i}^{\prime} .
$$

The lemma now follows by induction.

The first part of the next result for $n=2$ was found independently by W. D. Munn, and refinements (given below) were found by G. B. Preston.

THEOREM 5. Let $\rho$ be a congruence on a regular semigroup $S$. Let $A_{1}, A_{2}, \ldots, A_{n}$ be any elements of $S / \rho$ such that $A_{1} \mathscr{L} A_{2} \mathscr{L} \ldots \mathscr{L} A_{n}$ in $S / \rho$. Then there exist elements $a_{1}, a_{2}, \ldots, a_{n}$ in $S$ such that $a_{i} \in A_{i}(i=1,2, \ldots, n)$ and such that

(i) $a_{1} \mathscr{L} a_{2} \mathscr{L} \ldots \mathscr{L} a_{n}$

(ii) $a_{i}$ is an idempotent if $A_{i}$ is an idempotent of $S / \rho(i=1,2, \ldots, n)$.

Proof. For $i=1,2, \ldots, n$, let $A_{i}^{\prime}$ be an inverse of $A_{i}$ in $S / \rho$ but let $A_{i}^{\prime}=A_{i}$ if $A_{i}$ is an idempotent. Then, from Lemma 4 , for $i=1,2, \ldots, n$, there exist elements $x_{i} \in A_{i}$, $x_{i}^{\prime} \in A_{i}^{\prime} \cap V\left(x_{i}\right)$, such that $x_{1} \mathscr{L} x_{2} \mathscr{L} \ldots \mathscr{L} x_{n}$. For $i=1,2, \ldots, n$, if $A_{i}$ is an idempotent, then put $a_{i}=x_{i}^{\prime} x_{i} \in A_{t}^{\prime} A_{i}=A_{i}$, otherwise put $a_{i}=x_{i}$. Then $a_{1} \mathscr{L} a_{2} \mathscr{L} \ldots \mathscr{L} a_{n}, a_{i} \in A_{i}$, and $a_{i}^{2}=a_{i}$ if $A_{i}^{2}=A_{i}(i=1,2, \ldots, n)$. This proves the theorem.

The refinements mentioned above are as follows.

(i) For any elements $x \in A_{1}, y \in A_{2}$ there exists an idempotent $e \in S$ such that $x e \in A_{1}, y e \in A_{2}$ and $x e \mathscr{L}$ ye; 
(ii) for any elements $x_{i} \in A_{i}(i=1,2, \ldots, n)$ there exist elements $y_{i}(i=1,2, \ldots, n)$ such that $x_{i} y_{i} \in A_{i}(i=1,2, \ldots, n)$ and $x_{1} y_{1} \mathscr{L} x_{2} y_{2} \mathscr{L} \ldots \mathscr{L} x_{n} y_{n}$.

COROLlaRY 6. If $m$ is a positive integer and $S$ is a regular semigroup such that each $\mathscr{L}$-class of $S$ contains at most m elements [idempotents, $\mathscr{H}$-classes], then the same is true of each homomorphic image of $S$.

In particular, we have again the first statement of Theorem 7.36 of [1].

Definition (essentially from [6]). Let $\mathscr{E}$ be any relation on a semigroup $S$ and let $E$ be the set of idempotents of $S$. Then $S$ is called $\mathscr{E}$-regular if, for every congruence $\rho$ on $S$, $\rho \cap(E \times E) \subseteq \mathscr{E}$ implies $\rho \subseteq \mathscr{E}$.

THEOREM 7. Let $\mathscr{E}$ be any transitive relation containing $\mathscr{L}$ on a regular semigroup $S$. 'Then $S$ is $\mathscr{E}$-regular.

Proof. Let $E$ be the set of idempotents of $S$ and let $\rho$ be any congruence on $S$ such that $\rho \cap(E \times E) \subseteq \mathscr{E}$. Take any pair $(a, b) \in \rho$ and any inverses $a^{\prime}$ of $a$ and $b^{\prime}$ of $b$ in $S$. Then $a^{\prime} \rho$ and $b^{\prime} \rho$ are both inverses of $a \rho=b \rho$ in $S / \rho$, whence $\left(a^{\prime} a\right) \rho$ and $\left(b^{\prime} b\right) \rho$ are $\mathscr{L}$-related idempotents of $S / \rho$. By Theorem 5 there exist $\mathscr{L}$-related idempotents $e, f$ in $S$ such that $e \rho a^{\prime} a$, $f \rho b^{\prime} b$. . Then $a^{\prime} a \mathscr{E} e, f \mathscr{E} b^{\prime} b$ and further $a \mathscr{L} a^{\prime} a \mathscr{E} e \mathscr{L} f \mathscr{E} b^{\prime} b \mathscr{L} b$ and so $(a, b) \in \mathscr{E}$, giving $\rho \subseteq \mathscr{E}$.. Hence $S$ is $\mathscr{E}$-regular.

COROLlaRY 8. (This is Theorem 2.3 of [6].) Let $\mathscr{K}$ be any one of Green's relations on a regular semigroup $S$. Then $S$ is $\mathscr{K}$-regular.

Proof. Green's relations $\mathscr{L}, \mathscr{D}$ and $\mathscr{J}$ are of course transitive relations containing $\mathscr{L}$; so the corollary holds for them. The result for $\mathscr{R}$ is dual to the result for $\mathscr{L}$ and the result for $\mathscr{H}$ is an easy corollary of these two results. It is just as easy to deduce Theorem 1 of [2] as a corollary also.

\section{Green's relation $\mathscr{D}$.}

Lemma 9. Let $\rho$ be a congruence on a regular semigroup $S$. Take any positive integers $n, m$, and for $i=1,2, \ldots, n, j=1,2, \ldots, m$, let $A_{i}, A_{i}^{\prime}, B_{j}, C_{j}$ be elements of $S / \rho$ such that, for all $i$ and $j, A_{i} \mathscr{L} B_{j}$ (i.e. $A_{1} \mathscr{L} \ldots \mathscr{L} A_{n} \mathscr{L} B_{1} \ldots \mathscr{L} B_{m}$ ), $A_{i}^{\prime} \mathscr{V} A_{i}$ and $B_{j} \mathscr{R} C_{j}$ in $S / \rho$. Then, for each $i$ and $j$, there exist elements $a_{i} \in A_{i}, b_{j} \in B_{j}, a_{i}^{\prime} \in A_{i}^{\prime}, c_{j} \in C_{j}$ such that $a_{i} \mathscr{L} b_{j}, a_{i}^{\prime} \in V\left(a_{i}\right)$, and $b_{j} \mathscr{R} c_{j}$ in $S$, for all $i$ and $j$.

Proof. From Lemma 4, for $i=1,2, \ldots, n$, there exist $x_{i} \in A_{i}, x_{i}^{\prime} \in A_{i}^{\prime}$ such that $x_{i}^{\prime} \in V\left(x_{i}\right)$ and such that $x_{1} \mathscr{L} x_{2} \mathscr{L} \ldots \mathscr{L} x_{n}$. From the result dual to Lemma 4 there exist, for $j=1,2, \ldots, m$, $y_{j} \in B_{j}$ and $z_{j} \in C_{j}$ such that $y_{j} \mathscr{R} z_{j}$. For each $j$, let $y_{j}^{\prime}$ be an inverse of $y_{j}$ in $S$ and let $v_{j}$ be an element from $S^{1}$ such that $z_{j} v_{j}=y_{j}$. Let $w$ be any inverse of $x_{1}^{\prime} x_{1}\left[\left(y_{1}^{\prime} y_{1}\right)\left(y_{2}^{\prime} y_{2}\right) \ldots\left(y_{m}^{\prime} y_{m}\right)\right]$. For $i=1,2, \ldots, n, j=1,2, \ldots, m$, put

$$
\begin{aligned}
& a_{i}=x_{i}\left(y_{1}^{\prime} y_{1}\right)\left(y_{2}^{\prime} y_{2}\right) \ldots\left(y_{m}^{\prime} y_{m}\right) w\left(x_{1}^{\prime} x_{1}\right), \quad a_{i}^{\prime}=x_{i}^{\prime} a_{i} x_{i}^{\prime} . \\
& b_{j}=y_{j}\left(y_{j+1}^{\prime} y_{j+1}\right)\left(y_{j+2}^{\prime} y_{j+2}\right) \ldots\left(y_{m}^{\prime} y_{m}\right) w\left(x_{1}^{\prime} x_{1}\right), \\
& c_{j}=b_{j}\left(y_{1}^{\prime} y_{1}\right)\left(y_{2}^{\prime} y_{2}\right) \ldots\left(y_{j-1}^{\prime} y_{j-1}\right) y_{j}^{\prime} z_{j} .
\end{aligned}
$$


It is routine to show that $y_{j-1} y_{j}^{\prime} b_{j}=b_{j-1}(j=2,3, \ldots, m), x_{n} y_{1}^{\prime} b_{1}=a_{n}, x_{i-1} x_{i}^{\prime} a_{i}=$ $a_{i-1}(i=2,3, \ldots, n)$ (Result 2), $y_{m} w x_{1}^{\prime} a_{1}=b_{m}$, and finally that

$$
c_{j} v_{j}\left(y_{j+1}^{\prime} y_{j+1}\right) \ldots\left(y_{m}^{\prime} y_{m}\right) w x_{1}^{\prime} x_{1}=b_{j} \quad(j=1,2, \ldots, m) \text {. }
$$

We thus have, for each $i$ and $j$, that $a_{i} \mathscr{L} b_{j}$ and $b_{j} \mathscr{R} c_{j}$; and further, it is routine to show that $a_{i}^{\prime} \in V\left(a_{i}\right)$.

Denote $w \rho$ by $W$, an inverse of $A_{1}^{\prime} A_{1} B_{1}^{\prime} B_{1} B_{2}^{\prime} B_{2} \ldots B_{m}^{\prime} B_{m}=A_{1}^{\prime} A_{1}$ (Result 2). Then, for each $i$ and $j$,

$$
\begin{aligned}
a_{l} \in A_{i}\left(B_{1}^{\prime} B_{1}\right)\left(B_{2}^{\prime} B_{2}\right) \ldots\left(B_{m}^{\prime} B_{m}\right) W A_{1}^{\prime} A_{1} & =A_{i} W A_{1}^{\prime} A_{1}=A_{i} A_{1}^{\prime} A_{1} W A_{1}^{\prime} A_{1}=A_{i} A_{1}^{\prime} A_{1}=A_{i}, \\
a_{i}^{\prime} & =x_{i}^{\prime} a_{i} x_{i}^{\prime} \in A_{i}^{\prime} A_{i} A_{i}^{\prime}=A_{i}^{\prime}, \\
b_{j} \in B_{j}\left(B_{j+1}^{\prime} B_{j+1}\right) \ldots\left(B_{m}^{\prime} B_{m}\right) W A_{1}^{\prime} A_{1} & =B_{j} W A_{1}^{\prime} A_{1}=B_{j}, \\
c_{j} \in B_{j} B_{1}^{\prime} B_{1} \ldots B_{j-1}^{\prime} B_{j-1} B_{j}^{\prime} C_{j} & =B_{j} B_{j}^{\prime} C_{j}=C_{j} .
\end{aligned}
$$

This proves the lemma.

In general, if we take elements $B_{1}, B_{2}, C_{1}, C_{2}$ in $S / \rho$ such that $B_{1} \mathscr{L} B_{2} \mathscr{R} C_{2}$ and $B_{1} \mathscr{R} C_{1} \mathscr{L} C_{2}$ in $S / \rho$, then there are not necessarily elements $b_{1}, b_{2}, c_{1}, c_{2}$ in $B_{1}, B_{2}, C_{1}, C_{2}$ respectively, such that $b_{1} \mathscr{L} b_{2} \mathscr{R} c_{2}$ and $b_{1} \mathscr{R} c_{1} \mathscr{L} c_{2}$ in $S$.

THEOREM 10. Let $\rho$ be a congruence on a regular semigroup $S$. Let $A_{1}, A_{2}, \ldots, A_{n}$ be any elements of $S / \rho$ such that $A_{1} \mathscr{D} A_{2} \mathscr{D} \ldots \mathscr{D} A_{n}$ in $S / \rho$. Then there exist elements $a_{1}, a_{2}, \ldots, a_{n}$ in $S$ such that $a_{i} \in A_{i}(i=1,2, \ldots, n)$ and such that

(i) $a_{1} \mathscr{D} a_{2} \mathscr{D} \ldots \mathscr{D} a_{n}$

(ii) $a_{i}$ is an idempotent of $S$ if $A_{i}$ is an idempotent of $S / \rho(i=1,2, \ldots, n)$.

Proof. In the case in which there are no idempotents among $A_{1}, A_{2}, \ldots, A_{n}$ we may see that the theorem has been stated within Lemma 9 by simply regarding $C_{1}, C_{2}, \ldots, C_{m}$ in that lemma as any finite set of $\mathscr{D}$-related elements from $S / \rho$. However there is no loss of generality in assuming that at least one of $A_{1}, A_{2}, \ldots, A_{n}$ is an idempotent, since we could add an idempotent to this list if necessary. We may assume then, without loss of generality, that, for some integer $m$, where $1 \leqq m \leqq n, A_{1}, A_{2}, \ldots, A_{m}$ are all the idempotents from $A_{1}, A_{2}, \ldots, A_{n}$. For $i=1,2, \ldots, m$, take elements $B_{i}, B_{i}^{\prime}$ in $S / \rho$ such that $B_{i}^{\prime} \mathscr{V} B_{i}, B_{i}^{\prime} B_{i}=A_{1}$ and $B_{i} B_{i}^{\prime}=A_{i}$ [1, Section 2.3]. For $i=m+1, m+2, \ldots, n$ take an element $C_{i}$ in $S / \rho$ such that $A_{1} \mathscr{L} C_{i} \mathscr{R} A_{i}$. Then

$$
B_{1} \mathscr{L} B_{2} \mathscr{L} \ldots \mathscr{L} B_{m} \mathscr{L} C_{m+1} \mathscr{L} \ldots \mathscr{L} C_{n}
$$

From Lemma 9 there exist, for $i=1,2, \ldots, m, j=m+1, \ldots, n$, elements $b_{i} \in B_{i}, b_{i}^{\prime} \in B_{i}^{\prime}, c_{j} \in C_{j}$ and $a_{j} \in A_{j}$ such that $b_{i}^{\prime} \in V\left(b_{i}\right), c_{j} \mathscr{R} a_{j}$ (for all $i, j$ ) and such that

$$
b_{1} \mathscr{L} b_{2} \mathscr{L} \ldots \mathscr{L} b_{m} \mathscr{L} c_{m+1} \mathscr{L} \ldots \mathscr{L} c_{n} \text {. }
$$

For $i=1,2, \ldots, m$, put $a_{i}=b_{i} b_{i}^{\prime}$. Then $a_{1}, a_{2}, \ldots, a_{m}$ are idempotents, $a_{i} \in A_{i}(i=1,2, \ldots, n)$ and $a_{1} \mathscr{D} a_{2} \mathscr{D} \ldots \mathscr{D} a_{n}$, giving the theorem.

As a special case of Theorem 10 we obtain the first part of Corollary 34 of [10]. In fact 
it was this latter result and a discussion with G. B. Preston that brought to the author's attention the questions answered here. The author wishes to express his gratitude to Professor Preston for this and further helpful discussions.

COROLLARY 11. If $m$ is a positive integer and $S$ is a regular semigroup such that each $\mathscr{D}$-class of $S$ contains at most $m$ elements [idempotents, $\mathscr{R}$-classes, $\mathscr{H}$-classes], then the same is also true of each homomorphic image of $S$.

The statement concerning $\mathscr{R}$-classes is equivalent to the statement of Corollary 6 concerning $\mathscr{H}$-classes. Corollary 11 implies that, if $S$ is a regular semigroup satisfying any of the four conditions listed, then each homomorphic image of $S$ is completely semisimple. In the next section we shall see an example of a completely semisimple semigroup with a homomorphic image that is not completely semisimple.

For each relation $\rho$ on a semigroup $S$ we denote by $\rho^{*}$ the congruence on $S$ generated by $\rho$.

COROLlaRY 12. (This is Theorem 1.4 of [4].) The minimum semilattice congruence on a regular semigroup $S, \eta$ say, is determined by $\eta=\mathscr{D}^{*}=\mathscr{J}^{*}$.

Proof. On any semilattice, Green's relation $\mathscr{J}$ is trivial; thus for $S, \mathscr{J} \subseteq \eta$ and hence $\mathscr{J}^{*} \subseteq \eta$. Conversely, a regular semigroup $T$ is a semilattice if Green's relation $\mathscr{D}$ on $T$ is trivial, which we see as follows. Each element is an idempotent since each $\mathscr{D}(T)$-class contains an idempotent, and, since each $\mathscr{L}$-class and $\mathscr{R}$-class consists of a single idempotent, $T$ is an inverse semigroup of idempotents, i.e. a semilattice. By Theorem 10 , the semigroup $S / \mathscr{D}^{*}$ has Green's relation $\mathscr{D}$ trivial. Thus $\eta \subseteq \mathscr{D}^{*} \subseteq \mathscr{J}^{*}$, giving $\eta=\mathscr{D}^{*}=\mathscr{J}^{*}$ as required.

6. Some counterexamples. Let $N$ be the set of non-negative integers and let $B=N \times N$ be the bicyclic semigroup [1, Section 1.12]. Thus, for any $(m, n),(p, q)$ in $B,(m, n)(p, q)=$ $(m+p-\min (n, p), n+q-\min (n, p))$. Let $Z$ be the group of all integers under addition and let $\phi: B \rightarrow Z$ be the homomorphism that maps each $(m, n)$ in $B$ to $m-n$ in $Z$. Then $\phi$ is onto $Z$. Also $\mathscr{H}(Z)=Z \times Z, \mathscr{H}(B)=l_{B}$ (the identity relation on $B$ ) and so the question in the introduction has answer no for $\mathscr{K}=\mathscr{H}$.

Now put $S=\{[(m, n),(p, q)] \in B \times B: m+p=n+q\}$. Note that $B \times B$ is an inverse semigroup. It is routine to show that $S$ is a subsemigroup of $B \times B$, and clearly the inverse in $B \times B$ of each element in $S$ is also in $S$. Thus $S$ is an inverse semigroup. Note that $S$ contains all the idempotents of $B \times B$. Let $e, f$ be any distinct $\mathscr{D}$-related idempotents of $S$. Then there is an element $a$ in $S$ such that $a a^{-1}=e, a^{-1} a=f[1$, Section 2.3]. For some $m, n, p, q \in N$, we have $a=[(m, n),(p, q)]$ and then $a a^{-1}=[(m, n),(p, q)][(n, m),(q, p)]=$ $[(m, m),(p, p)]$, and $a^{-1} a=[(n, n),(q, q)]$. Since $m+p=n+q$, we have $m>n$ if and only if $p<q$, whence the two idempotents $e, f$ are not comparable. Thus $S$ is completely semisimple $[3$, Result 6$]$. Note that one of its homomorphic images, for example $B$, is not completely semisimple. Since $m+p=n+q$, we see that $D_{e}$ contains at most $m+p+1$ idempotents. Since $\mathscr{H}$ is trivial on $B$, on $B \times B$ and thus on $S, D_{e}$ contains at most $(m+p+1)^{2}$ elements. (It is easy to show that this is the exact number.) Thus each $\mathscr{D}$-class of $S$ contains finitely many elements (cf. Corollary 11).

Let $\psi$ be the homomorphism of $S$ onto $B$ such that $[(m, n),(p, q)] \psi=(m, n)$ for each 
element $[(m, n),(p, q)]$ in $S$. For any positive integer $d$ let us define, after Munn, $B_{d}=$ $\{(m, n) \in B: m \equiv n(\bmod d)\}$, a subsemigroup of $B$ that is a simple inverse $\omega$-semigroup containing $d \mathscr{D}$-classes [7, Example 1.4]. The $\mathscr{D}$-classes are given by

$$
D_{i}=\left\{(m, n) \in B_{d}: m \equiv i(\bmod d)\right\} \quad(0 \leqq i<d) .
$$

Now $B_{d} \psi^{-1}$ is an inverse subsemigroup of $S$ (since $S$ and $B_{d}$ are inverse semigroups) and $B_{d} \psi^{-1}$ is completely semisimple, since $S$ is completely semisimple, from [3, Result 6]. Thus $\mathscr{J}=\mathscr{D}$ on $B_{d} \psi^{-1}\left[1\right.$, Theorem 6.45]. But $\mathscr{J} \neq \mathscr{D}$ on $B_{d}$ if $d \geqq 2$. From this we see that the question in the introduction has answer no for $\mathscr{K}=\mathscr{J}$. We see this in more detail as follows: $(0,0) \mathscr{J}(1,1)$ in $B_{d}$ and there are no elements $a \in(0,0) \psi^{-1}$ and $b \in(1,1) \psi^{-1}$ such that $(a, b) \in \mathscr{J}=\mathscr{D}$ on $B_{d} \psi^{-1}$, since then we would have $(a \psi, b \psi) \in \mathscr{D}$ on $B_{d}$, i.e. $(0,0) \mathscr{D}(1,1)$ in $B_{\mathrm{d}}$, a contradiction.

From the semigroup $S$ and the homomorphism $\psi$ of $S$ onto $B$, we see that we cannot extend Theorem 5 [Theorem 10] to the case in which we consider a countably infinite set of elements from a single $\mathscr{L}$-class [ $\mathscr{D}$-class] of $S / \rho$ since each $\mathscr{D}$-class of $S$ is finite. Theorem 2 cannot be extended in this way either.

7. Other semigroups. For $S$ any finite semigroup the question in the introduction has been answered in [5, Proposition 3.2(b) and (d)]; only for $\mathscr{H}=\mathscr{H}$ does the question have answer no. The short proofs given in [5] are quite sufficient to prove the following slight generalizations:

(i) Let $\mathscr{K}$ be any of Green's relations $\mathscr{L}, \mathscr{R}, \mathscr{J}$ and let $M_{\mathrm{K}}$ be the corresponding minimal condition among $M_{L}, M_{R}, M_{J}[1$, Section 6.6]. Let $\rho$ be a congruence on a semigroup $S$ satisfying $M_{K}$ and let $K$ be any $\mathscr{K}$-class of $S / \rho$. Then there is a $\mathscr{K}$-class, $K^{\prime}$ say, of $S$, such that $K^{\prime} \rho$ औ $=K$.

(ii) Let $\rho$ be a congruence on a regular semigroup $S$ that satisfies $M_{L}$ (and hence $M_{R}, M_{J}$ $\left[1\right.$, Section 6.6]) and let $H$ be any $\mathscr{H}$-class of $S / \rho$. Then there is an $\mathscr{H}$-class, $H^{\prime}$ say, of $S$, such that $H^{\prime} \rho$ ' $=H$.

Since Green's relations are all trivial on any free semigroup, and any semigroup is a homomorphic image of a free semigroup, we see that all parts of the question in the introduction have answer no if we let $S$ be an arbitrary semigroup instead of a regular semigroup.

Before stating the next theorem we repeat some well-known [11, Section 3] and easilyproved results concerning equivalence relations on a set and congruences on a semigroup. Let $\sigma$ be any equivalence on a set $X$. For each equivalence $\gamma$ on $X$ containing $\sigma$ define $\gamma / \sigma=$ $\{(x \sigma, y \sigma):(x, y) \in \gamma\}$, an equivalence on $X / \sigma$. Any equivalence on $X / \sigma$ is of the form $\gamma / \sigma$ for some equivalence $\gamma$ on $X$ containing $\sigma$. Further, let $\gamma_{1}, \gamma_{2}$ be any equivalences on $X$ containing $\sigma$. Then $\gamma_{1} \subseteq \gamma_{2}$ if and only if $\gamma_{1} / \sigma \subseteq \gamma_{2} / \sigma$. If $X$ is a semigroup and $\sigma$ is a congruence, then $\gamma_{1}$ is a congruence on $X$ if and only if $\gamma_{1} / \sigma$ is a congruence on $X / \sigma$.

THEOREM 13. Let $\mathscr{K}$ be any one of Green's relations $\mathscr{H}, \mathscr{L}, \mathscr{R}, \mathscr{J}$. Let $\rho$ be a congruence contained in $\mathscr{K}$ on a semigroup $S$. Then $\mathscr{K}(S / \rho)=\mathscr{K}(S) / \rho$.

Proof. Let $\mathscr{K}=\mathscr{L}$. Take any elements $a, b$ in $S$ such that $a \rho$ and $b \rho$ are $\mathscr{L}$-related in 
$S / \rho$. Then either $a \rho \natural=b \rho$ औ (whence $(a, b) \in \rho \subseteq \mathscr{L}(S)$ ) or $a \rho \natural=u \rho \natural b \rho \natural=(u b) \rho \natural$ and $b \rho \natural=v \rho$ औ $a \rho \natural=(v a) \rho \natural$ for some elements $u, v \in S$. Then $(a, u b),(b, v a) \in \rho \subseteq \mathscr{L}(S)$, whence $a=x u b, b=y v a$ for some elements $x, y \in S$ and so $(a, b) \in \mathscr{L}(S)$. Thus $\mathscr{L}(S / \rho) \subseteq \mathscr{L}(S) / \rho$. But clearly $\mathscr{L}(S) / \rho \subseteq \mathscr{L}(S / \rho)$, giving $\mathscr{L}(S / \rho)=\mathscr{L}(S) / \rho$.

We deduce the result for $\mathscr{K}=\mathscr{R}$ dually and then that for $\mathscr{K}=\mathscr{H}$ as a corollary. The proof for $\mathscr{K}=\mathscr{J}$ is entirely similar to the proof for $\mathscr{K}=\mathscr{L}$.

The following result was communicated verbally to the author by W.D. Munn; the result for $\mathscr{K}=\mathscr{H}$ has been announced in [8, Theorem 2.1].

CoRollary 14. (Due to W. D. Munn.) Let $\mathscr{K}$ be any of $\mathscr{H}, \mathscr{L}, \mathscr{R}, \mathscr{J}$ and let $\rho$ be the maximum congruence contained in $\mathscr{K}$ on a semigroup $S$. Then there is no congruence on $S / \rho$ contained in $\mathscr{K}(S / \rho)$ except the trivial congruence.

Proof. Take any congruence on $S / \rho$ contained in $\mathscr{K}(S / \rho)$. Without loss of generality we may take this congruence to be $\gamma / \rho$, for some congruence $\gamma$ on $S$ containing $\rho$. Since $\gamma / \rho \subseteq \mathscr{K}(S / \rho)=\mathscr{K}(S) / \rho$, we have $\rho \subseteq \gamma \subseteq \mathscr{K}(S)$, whence $\rho=\gamma$ and $\gamma / \rho=\rho / \rho$ is the trivial congruence on $S / \rho$.

For $\mathscr{K}=\mathscr{D}$, neither Theorem 13 nor Corollary 14 is true, as can be seen from [1, Theorem 8.11 and Exercise 1, Section 8.2]. For $\mathscr{K}=\mathscr{D}$ and $S$ a regular semigroup, Theorem 13 and then Corollary 14 can be easily deduced from Theorem 10.

In [3] it is proved that, if $S$ is a regular semigroup containing at most $m$ idempotents in each $\mathscr{D}$-class (for some integer $m$ ), then, for each element $a \in S, a^{m}$ is an element of a subgroup of $S$. Although there is no mention of congruences in this result, the following theorem plays an important part in its proof.

THEOREM 15. Let $\rho$ be any congruence contained in $\mathscr{L}$ on a semigroup $S$. Then, for each pair $(a, b) \in \rho, H_{a}$ is a group if and only if $H_{b}$ is a group. If, further, $S$ is regular, then, for each element $a \in S, a$ is an element of a subgroup of $S$ if (and only if) a $a$ is an element of a subgroup of $S / \rho$.

Proof. Let $(a, b) \in \rho$ and suppose that $H_{a}$ is a group. Then $\left(a^{2}, b^{2}\right) \in \rho \subseteq \mathscr{L}$ and so $\left(b, b^{2}\right) \in \mathscr{L}$. To show that $H_{b}$ is a group, it is enough to prove that $\left(b, b^{2}\right) \in \mathscr{R}$ [1, Theorem 2.16].

Let $e$ denote the identity of $H_{a}$. Then $(e a, e b) \in \rho \subseteq \mathscr{L}$ and so $e b \in L_{e}$. Let $x$ be any inverse of $e b$ in $R_{e}$ and let $f=e b x e$. Then $f^{2}=f$. Also, if $a^{\prime}$ denotes the inverse of $a$ in $H_{e}$, we have that

$$
e=e a a^{\prime} \rho e b a^{\prime}=e b x e b a^{\prime} \rho e b x e a a^{\prime}=e b x e=f .
$$

Hence $(e, f) \in \mathscr{L}$. But $f \leqq e$ and so $f=e$. Thus

$$
b=b e=b(e b x e)=b^{2} x e
$$

and so $\left(b, b^{2}\right) \in \mathscr{R}$, as required. The first part of the theorem now follows. The author is very grateful to W. D. Munn for the above short proof.

Suppose now that $S$ is regular and take any element $a$ in $S$ such that $a \rho$ is a member of a subgroup of $S / \rho$. Let $(a \rho)^{*}$ be the inverse of $a \rho$ in this subgroup. By Lemma 1 there exist elements $x, x^{\prime} \in S$ such that $x^{\prime} \in V(x), x^{\prime} \in(a \rho)^{*}, x \in a \rho$. Then $x x^{\prime}, x^{\prime} x \in(a \rho)(a \rho)^{*}=(a \rho)^{*}(a \rho)$; 
so $\left(x x^{\prime}, x^{\prime} x\right) \in \rho \subseteq \mathscr{L}$ and then $x \mathscr{R} x x^{\prime}, x \mathscr{L} x^{\prime} x \mathscr{L} x x^{\prime}$, whence $x \mathscr{H} x x^{\prime}$ and $H_{x}$ is a group. But $(x, a) \in \rho$; so $H_{a}$ is a subgroup of $S$ from the above. Conversely, if $a$ is any element from a subgroup of $S$, then $a \mathscr{H} a^{2}$ in $S$, whence $a \rho \mathscr{H} a^{2} \rho=(a \rho)(a \rho)$ in $S / \rho$ and therefore $a \rho$ is an element of a subgroup of $S / \rho$.

\section{REFERENCES}

1. A. H. Clifford and G. B. Preston, The algebraic theory of semigroups, Amer. Math. Soc., Mathematical Surveys No. 7, Vols. I and II (Providence, R.I., 1961 and 1967).

2. T. E. Hall, On the lattice of congruences on a regular semigroup, Bull. Australian Math. Soc. 1 (1969), 231-235.

3. T. E. Hall, On regular semigroups, J. Algebra; to appear.

4. J. M. Howie and G. Lallement, Certain fundamental congruences on a regular semigroup, Proc. Glasgow Math. Assoc. 7 (1966), 145-159.

5. J. Rhodes, Some results on finite semigroups, J. Algebra 4 (1966), 471-504.

6. G. Lallement, Congruences et équivalences de Green sur un demi-groupe régulier, C.R. Acad. Sc. Paris, Série A, 262 (1966), 613-616.

7. W. D. Munn, Regular w-semigroups, Glasgow Math. J. 9 (1968), 46-66.

8. W. D. Munn, On simple inverse semigroups, Semigroup Forum 1 (1970), 63-74.

9. W. D. Munn, 0-bisimple inverse semigroups, J. Algebra 15 (1970), 570-588. 29-61.

10. G. B. Preston, Matrix representations of inverse semigroups, J. Australian Math. Soc. 9 (1969),

11. N. R. Reilly and H. E. Scheiblich, Congruences on regular semigroups, Pacific J. Math. 23 (1967), 349-360.

12. M. Yamada, Regular semigroups whose idempotents satisfy permutation identities, Pacific J. Math. 21 (1967), 371-392.

UNIVERSITY OF STIRLING 